\title{
Frailty among Older Adults Living in Nursing Homes in Indonesia: Prevalence and Associated Factors
}

\author{
Aulia Rizka ${ }^{1}$, Andika Indrarespati ${ }^{2}$, Noto Dwimartutie ${ }^{1}$, Muhadi Muhadi ${ }^{3}$ \\ ${ }^{1}$ Division of Geriatric Medicine, Department of Internal Medicine, Faculty of Medicine, Universitas Indonesia, Cipto Mangunkusumo National Hospital, Jakarta \\ Indonesia \\ ${ }^{2}$ Department of Internal Medicine, Faculty of Medicine, Universitas Indonesia, Cipto Mangunkusumo National Hospital, Jakarta, Indonesia \\ ${ }^{3}$ Division of Cardiovascular, Department of Internal Medicine, Faculty of Medicine, Universitas Indonesia, Cipto Mangunkusumo National Hospital, Jakarta, \\ Indonesia
}

\section{Corresponding Author:}

Aulia Rizka, MD, PhD

Department of Internal Medicine,

Faculty of Medicine, Universitas

Indonesia, Cipto Mangunkusumo

National General Hospital, Jakarta,

Indonesia

E-mail: dr.auliarizka@yahoo.co.id

ORCID:

https://orcid.org/0000-0002-6592-3354

Received: March 28, 2021

Revised: April 25, 2021

Accepted: May 7, 2021

\begin{abstract}
Background: Frailty syndrome is a predictor of all-cause mortality among older adults living in nursing homes. However, data on the prevalence of frailty among individuals living in nursing homes, particularly in middle-income countries, are limited. Thus, this study aimed to determine the prevalence of frailty and identify its associated factors among older adults living in nursing homes in Indonesia. Methods: This cross-sectional study of older adults living in six nursing homes in Indonesia was conducted between May and December 2019. Data on demographic characteristics, physical activity (Physical Activity Scale for the Elderly), cognitive status (Abbreviated Mental Test), nutritional status (Mini Nutritional Assessment Short-Form), depression (Geriatric Depression Scale), comorbidity, frailty state (Cardiovascular Health Study criteria), dietary pattern (24-hour food recall), handgrip strength, and gait speed were evaluated. Bivariate and multivariate analyses were performed to identify factors independently associated with frailty. Results: This study recruited a total of 214 participants with a mean age of $73.68 \pm 4.30$ years. The prevalence rates of frailty and malnutrition were $46.5 \%$ and $58 \%$, respectively. The results showed that physical frailty was associated with malnutrition (odds ratio $=4.23 ; 95 \%$ confidence interval, 1.730-10.380). Conclusion: Frailty was prevalent and strongly associated with malnutrition among older adults living in nursing homes in Indonesia.
\end{abstract}

Key Words: Older adult, Frailty syndrome, Nursing home

\section{INTRODUCTION}

Frailty significantly affects older adults. Frailty syndrome is characterized by decreased physiological reserves caused by multi-dimensional deficits associated with the aging process. Older adults with frailty are at an increased risk of falls and health issues, including disability, which are correlated with increased morbidity and mortality. ${ }^{1-4)}$ The prevalence of frailty ranges from $4.0 \%$ to $59.1 \%$, with an overall prevalence rate of $10.7 \%$. ${ }^{5)}$ A previous study conducted in Indonesia reported a frailty prevalence rate of $25.2 \%$ among older adults in the community ${ }^{6)}$ compared with $29 \%-$
87.5\% among individuals living in nursing homes in Western countries based on the frailty screening criteria. ${ }^{7)}$ Frailty is an important factor among older individuals living in nursing homes. A systematic review by Zhang et al. ${ }^{8)}$ showed that frailty was a predictor of all-cause mortality among older individuals living in nursing homes. Therefore, frailty screening and multi-disciplinary intervention strategies must be conducted immediately to prevent poor outcomes and reduce mortality rates among older individuals living in nursing homes.

The risk factors for frailty syndrome include aging, low physical activity, weight loss, poor nutrition, unhealthy lifestyle habits, poor 
living environment, comorbidities, polypharmacy, genetics, and female sex. These factors are interrelated, form a cycle, and cause conditions such as chronic malnutrition, inflammation, and disruption in hormone regulation and coagulation pathways. ${ }^{1)}$

In middle-income countries, including Indonesia, malnutrition and poor energy and protein intake are significant challenges among older adults. ${ }^{9,10)}$ Nursing homes in Indonesia are mostly funded by the government, private companies, and charity organizations. Moreover, older adults in Indonesia usually live with their children and grandchildren; thus, few older adults are sent by the family or are admitted to nursing homes. Usually, only older adults with many comorbidities and poor financial support move to nursing homes.

Previous studies have found that older individuals living in nursing homes present with cognitive impairment and depression. ${ }^{8)}$ Studies on the association between malnutrition and physical frailty among nursing home residents, particularly in Asia, where dietary patterns and leisure-time physical activities differ between countries, are limited. The development of intervention programs for older individuals requires the identification of important predictors of frailty. Therefore, this study aimed to determine the prevalence of frailty and identify its associated factors among older adults living in nursing homes in Indonesia.

\section{MATERIALS AND METHODS}

\section{Study Site and Cohort}

This cross-sectional study included 214 older individuals living in six nursing homes in Jakarta and Banten Provinces, Indonesia from May 2019 to July 2019. The inclusion criteria were nursing home residents aged $>60$ years who were willing to participate in the study. We excluded subjects with severe dependency or inability to walk or grab a dynamometer or who could not understand questionnaires owing to health problems. The Research Ethics Committee of Universitas Indonesia approved this study (No. KET1190/UN2.F1/ETIK/PPM.00.02/2019). Written informed consents were obtained from all subjects.

\section{Data Collection}

We collected data on demographic characteristics such as age, ethnicity, anthropometric measurements (including body weight and height estimated using knee height), body mass index (BMI), and the Comprehensive Geriatric Assessment domains. The participants were screened for depression using the 15 -item Geriatric Depression Scale, with scores $>10$ indicating depression. Malnutrition was assessed using the Mini Nutritional Assessment ShortForm, ${ }^{11)}$ with scores $<7$ indicating malnutrition. Cognitive status was assessed using the Abbreviated Mental Test (AMT), with scores $<8$ indicating cognitive impairment. Physical activity was measured using the Physical Activity Scale for the Elderly (PASE), with a cut-off score $>270$ suggesting normal physical activity. Functional status was evaluated using the Barthel Index for activities of daily living. Scores of 100, 61-99, and $\leq 60$ indicated independence, mild and moderate dependency, and severe dependency, respectively. We defined multimorbidity in this study as the presence of more than two comorbidities.

We defined frailty based on the Cardiovascular Health Study (CHS) criteria, ${ }^{12)}$ which comprises the following items: weight loss, weak grip strength, slow gait speed, exhaustion, and low energy expenditure. Frailty was defined as meeting three or more of the five criteria, while non-frailty was defined as meeting none to two criteria. Handgrip strength was assessed three times using a handgrip dynamometer (Jamar Hydraulic Hand Dynamometer Model J00105; Lafayette Instrument, Lafayette, Indiana) using the dominant hand at 30-minute intervals between each measurement. We recorded the highest value of the three measurements. Nutritionists performed a 24-hour food recall to calculate the daily total energy and protein intakes.

\section{Statistical Analysis}

We performed statistical analyses using IBM SPSS Statistics for Windows, version 20.0 (IBM, Armonk, NY, USA). We first assessed variables for homogeneity before performing the statistical analysis. Continuous variables are expressed as mean \pm standard deviation (SD), and categorical variables are expressed as frequencies and percentage. A bivariate analysis of categorical variables that might be related to frailty, such as sex, age, low physical activity, cognitive impairment, malnutrition, depression, multimorbidity, energy, and protein intake, was performed using chi-square tests. The multivariate analysis included variables with $\mathrm{p}$ values $<0.25$ in the bivariate analysis. We performed the multivariate analysis using logistic regression (backward method) to identify factors associated with frailty among nursing home residents. Variables with $\mathrm{p} \leq 0.05$ were considered statistically significant.

\section{RESULTS}

This study recruited a total of 214 participants with a mean age of $73.68 \pm 4.30$ years. Approximately $2 \%, 51.3 \%$, and $46.7 \%$ of participants were categorized as fit, pre-frail, and frail, respectively. The characteristics of the participants are listed in Table 1. Based on the results of the bivariate analysis, age, malnutrition, and depression were correlated with frailty syndrome (Table 2). The results of the final multivariate analysis (Table 3) showed that malnutrition was 
Table 1. Participants' characteristics

\begin{tabular}{|c|c|c|c|}
\hline Variable & All $(n=214)$ & Non-frail group $(n=115)$ & Frail group $(n=99)$ \\
\hline Sex, female & $152(71)$ & $65(56)$ & $89(89)$ \\
\hline Age (y) & $73.7 \pm 4.3$ & $77.1 \pm 7.9$ & $72.9 \pm 6.6$ \\
\hline Multimorbidity & $34(16.2)$ & $14(12)$ & $20(20)$ \\
\hline \multicolumn{4}{|l|}{ Comorbidity } \\
\hline Hypertension & $87(41.9)$ & $42(36)$ & $45(45)$ \\
\hline Diabetes mellitus & $45(21.9)$ & $15(13)$ & $30(30)$ \\
\hline Coronary artery disease & $30(14)$ & $10(8)$ & $20(20)$ \\
\hline Stroke & $10(5.7)$ & $6(5)$ & $4(4)$ \\
\hline \multicolumn{4}{|l|}{ Nutritional status } \\
\hline Malnutrition & $84(39)$ & $60(52)$ & $24(24)$ \\
\hline Normal & $126(58.8)$ & $38(33)$ & $88(88)$ \\
\hline $\operatorname{BMI}\left(\mathrm{kg} / \mathrm{m}^{2}\right)$ & $22.3 \pm 4.1$ & $22.6 \pm 3.5$ & $21.3 \pm 4.6$ \\
\hline \multicolumn{4}{|l|}{ Cognitive status } \\
\hline Suspected cognitive impairment & $46(21.4)$ & $26(22)$ & $20(20)$ \\
\hline Normal & $168(78)$ & $86(74.7)$ & $82(82.8)$ \\
\hline Depression or suspected & $44(20)$ & $30(26)$ & $14(14.1)$ \\
\hline \multicolumn{4}{|l|}{ Dependency state } \\
\hline Independent & $208(97)$ & $110(95)$ & $98(99)$ \\
\hline Mild to moderate dependency & $6(3)$ & $5(5)$ & $1(1)$ \\
\hline Handgrip strength (kg), & $16(2-40)$ & $19.4(14-23)$ & $12.9(8-17.1)$ \\
\hline Physical activity, PASE score (kcal/wk) & $170.0 \pm 13.5$ & $189.8 \pm 12.5$ & $152.4 \pm 9.0$ \\
\hline Walking speed $(\mathrm{m} / \mathrm{s})$ & $0.66 \pm 0.34$ & $0.84 \pm 0.30$ & $0.23 \pm 0.03$ \\
\hline Energy intake (kcal/day) & $1,245.3 \pm 72.0$ & $1,288.0 \pm 218.0$ & $1,062.0 \pm 315.0$ \\
\hline Protein intake (kg BW/day) & $36.1 \pm 18.0$ & $38.2 \pm 9.1$ & $32.2 \pm 11.1$ \\
\hline
\end{tabular}

Values are presented as number of participants (\%) or mean \pm standard deviation or median (range).

BMI, body mass index; PASE, Physical Activity Scale for the Elderly; BW, body weight.

Table 2. Bivariate analysis of factors associated with frailty syndrome

\begin{tabular}{lcc}
\hline Variable & OR $(95 \%$ CI $)$ & p-value \\
\hline Sex & $0.84(0.95-1.62)$ & 0.340 \\
Age $>70 y$ & $2.07(1.00-4.27)$ & 0.010 \\
Low physical activity & $3.87(0.61-24.52)$ & 0.067 \\
Cognitive impairment & $0.80(0.44-1.44)$ & 0.433 \\
Malnutrition & $2.28(1.49-3.51)$ & $<0.001$ \\
Depression & $1.72(1.16-2.53)$ & 0.017 \\
Multimorbidity & $1.46(0.90-2.27)$ & 0.236 \\
Energy intake & $1.45(0.80-3.31)$ & 0.310 \\
Protein intake & $2.15(0.50-4.60)$ & 0.280 \\
\hline
\end{tabular}

$\mathrm{OR}$, odds ratio; $\mathrm{CI}$, confidence interval.

associated with frailty syndrome among older individuals living in nursing homes.

\section{DISCUSSION}

Approximately $83.8 \%$ of participants had $<2$ comorbidities, similar to the results of a previous study on individuals in the commu-
Table 3. Multivariate analysis of factors associated with frailty syndrome

\begin{tabular}{llc}
\hline Variable & \multicolumn{1}{c}{ OR $(95 \% \mathrm{CI})$} & p-value \\
\hline Malnutrition & $4.23(1.73-10.38)$ & 0.002 \\
Older age & $2.83(0.80-2.96)$ & 0.080 \\
Depression & $8.30(0.78-87.40)$ & 0.070 \\
\hline
\end{tabular}

OR, odds ratio; CI, confidence interval.

nity that reported $\leq 2$ comorbidities in $77 \%$ of older adult participants in the community. ${ }^{6}$ Hypertension was the most common disease in the present study, a finding consistent with the $2013 \mathrm{Na}$ tional Basic Health Research data showing that hypertension was the most frequent health issue among older adult individuals in Indonesia. ${ }^{13)}$ Although the proportion of participants with comorbidities was relatively small, most had a low level of physical activity and reduced handgrip strength and walking speed. The level of physical activity among the participants was lower than that among older adults in the community (PASE score: 170 [range, 156.5$184 \mathrm{kcal} /$ week] vs. 322 [range, 42-2,704 kcal/week]). ${ }^{14)}$ Ismail et $\mathrm{al}^{15)}$ reported a higher physical activity level in an older adult population in Malaysia, with an average PASE score of $664.3 \mathrm{kcal} /$ 
week. The median handgrip strength of participants in the present study (16 kg [range, 2-40 kg]) was lower than the normal handgrip strength of older individuals according to the CHS criteria (which was in accordance with the BMI of female participants) and the Asian Working Group for Sarcopenia ( $\geq 18 \mathrm{~kg}){ }^{16)}$ The low walking speed in this population $(0.66 \mathrm{~m} / \mathrm{s})$ was even lower than that reported in a study in community $(0.85 \mathrm{~m} / \mathrm{s})^{14)}$ and outpatient $(0.85 \mathrm{~m} / \mathrm{s})$ settings. ${ }^{17)}$ A 4-year cohort study of 3,018 participants aged $>64$ years who lived in the community in China reported that women had a faster walking speed at $0.96(\mathrm{SD}=0.21)$ $\mathrm{m} / \mathrm{s}^{18)}$

Frailty is common among nursing home residents based on their low handgrip strength and walking speed. A study conducted in Bali, Indonesia reported a frailty prevalence rate of $43.3 \%$ compared with $69.3 \%$ and 55.6\%, respectively, among individuals in Spain $^{19)}$ and China. ${ }^{20)}$ However, the cohorts in these previous studies were significantly older than the cohort in the current study. We observed a significantly higher prevalence of frailty in a relatively younger population, a finding that requires further assessment to facilitate the early diagnosis and treatment of frailty.

We did not observe an association between age and frailty, contrary to the results of a study on older Indonesian community-dwelling individuals, in which age $>70$ years was significantly correlated with frailty (odds ratio $[\mathrm{OR}]=5.27$; 95\% confidence interval $[\mathrm{CI}], 2.92-9.52) .{ }^{16)}$ Along with the aging process, physiological changes such as the activation of certain inflammatory processes, changes in body composition (decreased fat-free muscle mass), hormonal imbalances, and insulin resistance, which can eventually contribute to the development of frailty syndrome, have also been observed among older adult individuals. ${ }^{4}$

However, we observed no relationship between low levels of physical activity and frailty syndrome, unlike the finding reported in the study by Peterson et al., ${ }^{21)}$ where participants with a low level of physical activity or sedentary lifestyle had higher risks of frailty $(\mathrm{OR}=1.45 ; 95 \% \mathrm{CI}, 1.04-2.01)$. The differences in study results can be attributed to the low physical activity level in most participants.

We observed no association between cognitive impairment and frailty syndrome in the present study, contrary to the report by Chen et al. ${ }^{22)}$ who showed that impaired cognitive function was independently associated with frailty syndrome $(\mathrm{OR}=2.73 ; 95 \%$ CI, 1.09-6.83). This discrepancy might be attributed to the fact that most participants had normal cognitive status and different instruments (AMT vs. Mini-Mental State Examination [MMSE]) were used. Moreover, the Singapore Longitudinal Aging Study showed differences in the proportions of participants with different frailty states who presented with cognitive decline (MMSE score <26). In particular, the pre-frail and frail older adult groups with cognitive impairment (older people with cognitive frailty, excluding dementia) had increased risks of disability, worse quality of life, and mortality. ${ }^{23)}$

The results of this study showed that malnutrition alone was associated with frailty. Malnutrition in the context of poor nutrition and obesity can be correlated with frailty syndrome by increasing the composition of fatty tissues in the body, which may further increase the production of pro-inflammatory cytokines such as interleukin-6 and C-reactive protein. Another study in Indonesia reported that older adults had low energy and protein intake. ${ }^{13)}$ Therefore, malnutrition was associated with frailty. However, these are two distinct entities. Both are reversible; thus, nursing home residents with malnutrition or pre-frailty and frailty should receive early treatment to prevent these conditions from worsening. As we excluded subjects who could not perform frailty tests or answer questionnaires, the limitation of this study is that the prevalence of frailty in this population could be under-reported.

In conclusion, a significant proportion of older adults living in nursing homes presented with frailty. Malnutrition was significantly associated with physical frailty. Therefore, periodic evaluations of the nutritional and frailty status of older adults living in nursing homes are needed to assess the development of malnutrition and frailty. Moreover, further studies are warranted to develop intervention programs for malnourished, pre-frail, and frail individuals living in nursing homes.

\section{ACKNOWLEDGMENTS}

\section{CONFLICT OF INTEREST}

The researchers claim no conflicts of interest.

\section{FUNDING}

This work was supported by Publikasi Terindeks Internasional (PUTI) grant from Universitas Indonesia.

\section{AUTHOR CONTRIBUTION}

Conceptualization, AR; Data curation, AI; Funding acquisition, AR; Investigation, AR, AI; Methodology, AR; Project administration, AI; Supervision, AR, ND, M; Writing-original draft, AI, AR, SSL; Writing-review \& editing, AR.

\section{REFERENCES}

1. Clegg A, Young J, Iliffe S, Rikkert MO, Rockwood K. Frailty in elderly people. Lancet 2013;381:752-62.

2. Kojima G. Frailty as a predictor of future falls among communi- 
ty-dwelling older people: a systematic review and meta-analysis. J Am Med Dir Assoc 2015;16:1027-33.

3. Kojima G, Iliffe S, Jivraj S, Walters K. Association between frailty and quality of life among community-dwelling older people: a systematic review and meta-analysis. J Epidemiol Community Health 2016;70:716-21.

4. Morley JE, Vellas B, van Kan GA, Anker SD, Bauer JM, Bernabei $\mathrm{R}$, et al. Frailty consensus: a call to action. J Am Med Dir Assoc 2013;14:392-7.

5. Collard RM, Boter H, Schoevers RA, Oude Voshaar RC. Prevalence of frailty in community-dwelling older persons: a systematic review.J Am Geriatr Soc 2012;60:1487-92.

6. Setiati S, Laksmi PW, Aryana IG, Sunarti S, Widajanti N, Dwipa $\mathrm{L}$, et al. Frailty state among Indonesian elderly: prevalence, associated factors, and frailty state transition. BMC Geriatr 2019; 19:182.

7. Kojima G. Prevalence of frailty in nursing homes: a systematic review and meta-analysis. J Am Med Dir Assoc 2015; 16:940-5.

8. Zhang X, Dou Q, Zhang W, Wang C, Xie X, Yang Y, et al. Frailty as a predictor of all-cause mortality among older nursing home residents: a systematic review and meta-analysis. J Am Med Dir Assoc 2019;20:657-663. e4.

9. Arjuna T, Soenen S, Hasnawati RA, Lange K, Chapman I, Luscombe-Marsh ND. A cross-sectional study of nutrient intake and health status among older adults in Yogyakarta Indonesia. Nutrients 2017;9:1240.

10. Setiati S, Harimurti K, Dewiasty E, Istanti R, Mupangati YM, Ngestiningsih D, et al. Profile of food and nutrient intake among Indonesian elderly population and factors associated with energy intake: a multi-centre study. Acta Med Indones 2013;45:26574.

11. Kaiser MJ, Bauer JM, Ramsch C, Uter W, Guigoz Y, Cederholm T, et al. Validation of the Mini Nutritional Assessment shortform (MNA-SF): a practical tool for identification of nutritional status. J Nutr Health Aging 2009; 13:782-8.

12. Fried LP, Tangen CM, Walston J, Newman AB, Hirsch C, Gottdiener J, et al. Frailty in older adults: evidence for a phenotype. J Gerontol A Biol Sci Med Sci 2001;56:M146-56.

13. Ministry of Health of the Republic of Indonesia. RISKESDAS 2013: basic health research [Internet]. Jakarta, Indonesia: Ministry of Health; c2021 [cited 2021 May 18]. Available from: https://labmandat.litbang.kemkes.go.id/riset-badan-litbangkes/ menu-riskesnas/menu-riskesdas/374-rkd-2013.

14. Rensa R, Setiati S, Laksmi PW, Rinaldi I. Factors associated with physical frailty in elderly women with low socioeconomic status in urban communities: a cross-sectional study. Acta Med Indones 2019;51:220-9.

15. Ismail N, Hairi F, Choo WY, Hairi NN, Peramalah D, Bulgiba A. The Physical Activity Scale for the Elderly (PASE): validity and reliability among community-dwelling older adults in Malaysia. Asia Pac J Public Health 2015;27(8 Suppl):62S-72S.

16. Chen LK, Liu LK, Woo J, Assantachai P, Auyeung TW, Bahyah KS, et al. Sarcopenia in Asia: consensus report of the Asian Working Group for Sarcopenia. J Am Med Dir Assoc 2014;15: 95-101.

17. Seto E, Setiati S, Laksmi PW, Tamin TZ. Diagnostic test of a scoring system for frailty syndrome in the elderly according to cardiovascular health study, study of osteoporotic fracture and comprehensive geriatric assessment based frailty index compared with frailty index 40 items. Acta Med Indones 2015; 47:183-7.

18. Lee JS, Auyeung TW, Leung J, Kwok T, Woo J. Transitions in frailty states among community-living older adults and their associated factors. J Am Med Dir Assoc 2014;15:281-6.

19. de la Rica-Escuin M, Gonzalez-Vaca J, Varela-Perez R, Arjonilla-Garcia MD, Silva-Iglesias M, Oliver-Carbonell JL, et al. Frailty and mortality or incident disability in institutionalized older adults: the FINAL study. Maturitas 2014;78:329-34.

20. Liu W, Puts M, Jiang F, Zhou C, Tang S, Chen S. Physical frailty and its associated factors among elderly nursing home residents in China. BMC Geriatr 2020;20:294.

21. Peterson MJ, Giuliani C, Morey MC, Pieper CF, Evenson KR, Mercer V, et al. Physical activity as a preventative factor for frailty: the health, aging, and body composition study. J Gerontol A Biol Sci Med Sci 2009;64:61-8.

22. Chen S, Honda T, Chen T, Narazaki K, Haeuchi Y, Supartini A, et al. Screening for frailty phenotype with objectively-measured physical activity in a west Japanese suburban community: evidence from the Sasaguri Genkimon Study. BMC Geriatr 2015; 15:36.

23. Feng L, Zin Nyunt MS, Gao Q, Feng L, Yap KB, Ng TP. Cognitive frailty and adverse health outcomes: findings from the Singapore Longitudinal Ageing Studies (SLAS). J Am Med Dir Assoc $2017 ; 18: 252-8$. 